\title{
Osteoporosis asociada a indinavir en paciente infectado por virus de inmunodeficiencia humana. Comunicación de un caso y revisión de la literatura
}

\author{
MARTÍN LASSO B. ${ }^{1}$, GILBERTO GONZÁLEZ V. ${ }^{2}$, JORGE PÉREZ G. ${ }^{1}$, \\ LUIS M NORIEGA R. ${ }^{1}$ y ANDREA MALEBRAN R. ${ }^{3}$
}

\section{Osteoporosis associated to indinavir in an HIV infected patient. Case report and review}

The case of a 45 year-old HIV patient that developed secondary osteoporosis to 33 months of intake indinavir sulfate as part of its high activity antiretroviral therapy (HAART) is presented. His management consisted on alendronate, calcium and vitamin D together with modification of his antiretroviral therapy: indinavir moved to efavirenz. After 16 months of treatment an increment on the average in the bone mineral density of $11 \%$ was verified. During this time there were neither fractures, nor adverse effects or medication interactions. The literature is reviewed.

Key words: Osteoporosis, Indinavir, Alendronate, HIV.

\section{Introducción}

A raíz del advenimiento de terapias efectivas contra el VIH, que deben ser administradas por tiempo indefinido, nos hemos enfrentado a diversos efectos adversos relacionados al uso prolongado de estos fármacos: neuropatía periférica, pancreatitis, trastornos hematológicos, dislipidemia, lipodistrofia, resistencia insulínica, acidosis láctica y litiasis renal, entre los más importantes. Por otro lado, se ha comunicado la pérdida ósea significativa asociada al uso de inhibidores de proteasa como parte de la terapia antiretroviral $(\mathrm{TAR})^{1}$. A continuación presentamos un caso clínico de osteoporosis asociada a indinavir.

\section{Caso Clínico}

Hombre de 45 años, portador de VIH diagnosticado en 1991, sin otros antecedentes mórbidos de importancia. Inició TAR en enero de 1998 encontrándose en ese momento en etapa A2 de su enfermedad, con un recuento de linfocitos CD4 (L-CD4) de 201/ $\mathrm{mm}^{3}$ y una carga viral de 57.581 copias ARN/ml. La TAR consistió en: zidovudina $600 \mathrm{mg} /$ día, lamivudina $300 \mathrm{mg} /$ día e indinavir $2.400 \mathrm{mg} /$ día. Sus exámenes de laboratorio de ese momento no mostraban alteraciones y la radiografía de tórax tenía estructuras óseas normales. El paciente se caracterizó por excelente adherencia a su tratamiento incrementando su nivel de L-CD4 hasta 390 células/mm $3 \mathrm{~m}^{3}$ y logrando cargas virales indetectables en todos sus controles (6 en total) hasta marzo del año 2000. Durante su evolución tuvo discreta lipodistrofia y aumento de triglicéridos hasta $430 \mathrm{mg} / \mathrm{dl}$. Catorce meses luego de iniciado el TAR presentó un episodio de hematuria y dolor escrotal autolimitado con una ecografía testicular normal. A partir de los 22 meses de iniciada la terapia refirió dolor leve de caderas que se manejó con antiinflamatorios no esteroidales cediendo inicialmente para después volverse constante a partir de los

\footnotetext{
Unidad de Infectología. Hospital Dr. Sótero Del Río.

Unidad de Endocrinología Hospital Dr. Sótero Del Río y Hospital Clínico Pontificia Universidad Católica de Chile.

Enfermera Universitaria. Corporación de Investigaciones Médicas. Servicio de Salud Metropolitano Sur Oriente.
} 
28 meses de tratamiento. Radiografías de pelvis y columna lumbar efectuadas al mes 31 de TAR revelaron imágenes de mineralización ósea disminuida en forma difusa tanto lumbar como pélvica (Figura 1). El mes 33 de TAR se le efectuó una densitometría ósea con doble haz de rayos $\mathrm{X}$ que demostró una densidad mineral ósea (DMO) de la columna lumbar a nivel L2-L4 de $0,744 \mathrm{~g} / \mathrm{cm}^{2}$ ubicándose 3,79 DS por debajo del promedio para su edad; además en el cuello femoral izquierdo una densidad mineral ósea de $0,690 \mathrm{~g} / \mathrm{cm}^{2}$ ubicándose 2,46 DS por debajo del promedio para su edad. Se concluyó que existía osteoporosis de la columna lumbar y osteopenia severa de la cadera izquierda.

Su estudio en ese momento mostró un hemograma y perfil bioquímico completo (incluyendo calcio y fósforo) normales, excepto una fosfatasa alcalina de $132 \mathrm{U} / \mathrm{L}$, colesterolemia de $266 \mathrm{mg} / \mathrm{dl}$ y trgliceridemia de $619 \mathrm{mg} / \mathrm{dl}$. Tenía además un clearence de creatinina de $84,9 \mathrm{ml} / \mathrm{min}$, PTH: 45 $\mathrm{pg} / \mathrm{mL}$, TSH: $2,57 \mathrm{uUI} / \mathrm{mL}$, testosterona total: $419 \mathrm{ng} / \mathrm{dL}$ y calciuria de $24 \mathrm{~h}: 249 \mathrm{mg}$. Se decidió suspender indinavir e iniciar efavirenz $300 \mathrm{mg} / \mathrm{día}$ asociado a los otros dos antiretrovirales que ya recibía. Se indicó calcio, vitamina $\mathrm{D}$ y alendronato $10 \mathrm{mg} /$ día, terapia que mantiene hasta la actualidad sin complicaciones relacionadas. Una densitometría ósea realizada 16 meses después de la suspensión de indinavir demostró una DMO de columna lumbar L2-L4 de 0,857 g/ $\mathrm{cm}^{2}, 3,09$ DS bajo el promedio para su edad, y en el cuello femoral izquierdo una DMO de $0,767 \mathrm{~g} / \mathrm{cm}^{2}$, 1,75 DS bajo el promedio referencial. Al comparar con la densitometría inicial existía un incremento de $10,7 \%$ en la región lumbar y de $11,2 \%$ en el cuello femoral. Al momento actual el paciente permanece estable con carga viral de 1.500 copias ARN /ml y L-CD4 $331 \mathrm{cel} / \mathrm{mm}^{3}$.

\section{Discusión}

En los hombres ocurre osteoporosis primaria asociada a la mayor edad, pero un importante número presenta osteoporosis secundaria a trastornos endocrinológicos como el hipogonadismo, hipertiroidismo, hiperparatiroidismo, enfermedades hematológicas, enfermedades del tejido conectivo, trastornos renales, inmunopatías, enfermedad inflamatoria intestinal y cierto fármacos como los glucocorticoides, heparina, anticonvulsivantes, metotrexato, ciclosporina, antiácidos con aluminio y análogos de GnRH².

La asociación de infección por VIH y pérdida de masa ósea no es nueva ${ }^{3,4}$. Se postula que la

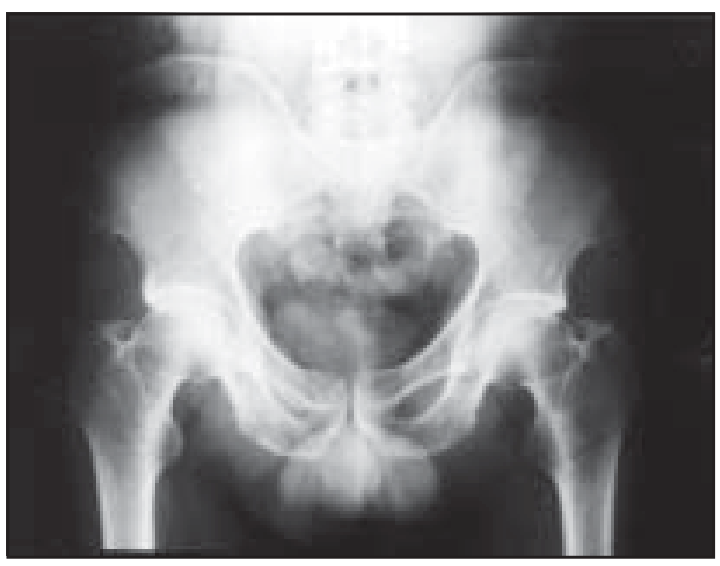

Figura 1. Radiografía de pelvis de paciente con osteoporosis asociada a indinavir. Nótese la importante pérdida ósea en el cuello femoral y trocánteres.

infección por VIH puede generar pérdida ósea secundaria a la actividad persistente de citoquinas, fundamentalmente el complejo factor de necrosis tumoral alfa (FNT $\alpha$ ) y las interleukinas (IL), especialmente la IL-1; estos mediadores inflamatorios pueden estimular la actividad osteoclástica y promover la reabsorción ósea como también tendrían un rol inhibiendo la actividad osteoblástica ${ }^{5}$. Otro factor de pérdida ósea en la infección por VIH estaría dado por un déficit severo de 1,25-dihidroxi-vitamina D3 descrito en estos pacientes y que ocurriría a raíz de la alteración de la 1-hidroxilasa renal por un efecto inhibidor del FNT $\alpha^{6}$.

El hipogonadismo ha sido descrito en 29 a $50 \%$ de los pacientes con $\operatorname{SIDA}^{7,8}$ y también es un factor a considerar. Se ha correlacionado también el bajo peso y acidosis láctica ${ }^{9}$, el antecedente de neumonía por Pneumocystis jirovecii (ex carinii) y el tratamiento corticoesteroidal como factores de riesgo para complicaciones asociadas a pérdida ósea ${ }^{10,11}$. Ninguno de estos factores fue detectado en nuestro paciente. Se ha postulado además que la infección por VIH per se estaría involucrada en la pérdida de hueso, Paton et al estudiaron prospectivamente, por un tiempo promedio de 15 meses, a 45 pacientes infectados por VIH sin TAR encontrando una disminución leve no significativa de la DMO en este grupo, respecto a su control ${ }^{12}$.

Recientemente se pensaba que la ¿TAR? podría favorecer la remodelación ósea y prevenir una posible osteoporosis asociada a la infección por $\mathrm{VIH}^{10}$; sin embargo, en el último tiempo han sido notificadas dos series de pacientes con osteoporosis relacionada al ¿TAR? y especialmente a aquellos esquemas que incluyen inhibi- 
dores de proteasa (IP) como el sulfato de indinavir, detectándose una incidencia $28,4 \%$ de osteopenia y 9,5 a $21 \%$ de osteoporosis severa ${ }^{1,13}$.

El mecanismo de pérdida ósea asociada a IP no está claro, aparentemente no guarda correlación con la lipodistrofia y pareciera ser que también existe un incremento en el recambio óseo con aumento de la reabsorción, en desmedro de la formación de hueso debido a la presencia de hipercalciuria, incremento de la fracción ósea de la ¿FA? y de la piridolina urinaria ${ }^{14}$. Además los IP favorecerían la actividad de la citocromo P 450 promoviendo la actividad enzimática de la 1hidrolasa lo que influye en el metabolismo de la vitamina $\mathrm{D}^{15}$.

Como no está dilucidada aún la fisiopatología de la pérdida de masa ósea en el usuario de IP, su tratamiento tampoco es claro. Se demostró que luego de 24 semanas de sustitución de IP por análogos nucleósidos y no nucleósidos inhibidores de la transcriptasa reversa en pacientes infectados con VIH, con osteoporosis y carga viral indetectable, no existió modificación en su $\mathrm{DMO}^{16}$. Por otro lado, el efecto benéfico del alendronato en pacientes hombres no infectados por VIH con osteoporosis, ha sido demostrado claramente, decribiéndose incremento en la DMO de $7,1 \%$ en la región lumbar y 2,5\% en el cuello femoral, luego de dos años de tratamiento ${ }^{17}$. Además recientemente se comunicó un caso de tratamiento de osteoporosis con alendronato en un paciente hombre infectado por VIH con un incremento en la DMO de 20,4\% en 6 meses pero que debió ser suspendido, incluyendo su TAR con indinavir, por la aparición de acidosis láctica ${ }^{18}$. En suma, pensamos que el manejo de estas complicaciones debe ser enfrentado por un equipo multidisciplinario, con un control muy próximo del paciente y que el efecto real del alendronato y de la suspensión de IP en pacientes infectados por VIH con osteoporosis, debe ser objeto de un estudio amplio y controlado.

\section{Resumen}

Se presenta el caso de un paciente de 45 años portador de VIH que desarrolló osteoporosis secundaria a 33 meses de exposición a sulfato de indinavir como parte de su terapia antiretroviral (TAR). Su manejo consistió en alendronato, calcio y vitamina D junto con modificación de su TAR: suspensión de indinavir e inicio de efavirenz. Luego de 16 meses de tratamiento se verificó un incremento en la densidad mineral ósea de $11 \%$ en promedio. No ocurrieron fracturas ni se constató efectos adversos o interacciones medicamentosas. Se revisa la literatura.

\section{Bibliografía}

1.- Tebas P, Powderly W G, Claxton S, Marin D, Tantisiriwat W, Teiltelbaum S L et al. Acelerated bone mineral loss in HIV-infected patients receiving potent antiretroviral therapy. AIDS 2000; 14 (4): F63-7.

2.- Campusano C. Enfrentamiento clínico del paciente con osteoporosis. En: Osteoporosis para médicos no especialistas. Boletín de la Escuela de Medicina. Pontificia Universidad Católica de Chile 1999; 28 (12): 24-30.

3.- Quero J H, Centeno N O, Munoz-Torres M. Alterations in bone turnover in HIV-positive patients. Infection 1993; 21: 220-2.

4.- Serrano S, Marinoso M L, Soriano J C. Bone remodelling in Human Immunodeficiency Virus 1 infected patients. A hystomorphometric study. Bone 1995; 16: 185-91.

5.- Aukrust P, Haug C J, Ueland T, Lien E, Müller F, Espevik $\mathrm{T}$ et al. Decreased bone formative an enhanced reasorptive markers in Human Immunodeficiency Virus infection: Indication of normalization of the bone remodeling process during Highly Active Antiretroviral Therapy. J Clin Endocrinol Metab 1999; 84 (1): 145-50.

6.- Haug C J, Aukrust P, Haug E, Morkrid L, Múller F, Froland S S. Severe deficiency of 1,25-Dihydroxyvitamin D3 in Human Immunodeficiency Virus infection: Association with immunological hyperactivity and only minor changes in calcium homeostasis. J Clin Endocrinol Metab 1998; 83 (11): 3832-8.

7.- Dobs A S, Dempsey M A, Ladenson P W, Polk B F. Endocrine disorders in men infected with Human Immunodeficiency Virus. Am J Med 1988; 84: 611-6.

8.- Raffi F, Brisseau J, Planchon B, Remi J, Barrier J H, Grolleau J. Endocrine function in 98 HIV-infected patients: a prospective study. AIDS; 1991; 5: 729-33.

9.- Carr A, Miller J, Cooper D. Osteopenia in HIV-infected men: association with lactic acidemia and lower weight pre-antiretroviral therapy. Antiviral Ther 2000; 5: S1520.

10.- Glesby M, Vaamonde C. Case-control study of a vascular necrosis in HIV-infected patients. Antiviral Ther 2000; 5: S15: 21.

11.- Masur H, Miller K D, Jones E C et al. High prevalence of avascular necrosis of the hip in HIV infection. Program and Abstracts of the $38^{\text {th }}$ Annual Meeting of the Infectious Diseases Society of American; September 7-10, 2000; New Orleans, Lousiana. (abstract $15)$.

12.- Paton N, Macallan D, Griffin G, Paisanas M. Bone mineral density in patients with Human Immunodeficiency Virus Infection. Calcif Tissue Int 1997; 61: 30-2.

13.- Hoy J, Hudson J, Law M, Cooper D A. Osteopenia in a randomized, multicenter study of protease inhibitor (PI) substitution in patients with lypodystrophy syndrome and well-controlled HIV viremia. Program and Abstracts $7^{\text {th }}$ Conference on Retroviruses and Opportunistic Infections. San Francisco California. [abstract 208]. www.retroconference.org

14.- Tebas P, Yarasheski K, Whyte M, Claxton S, DeMarco D, Powderly W. Serum and urine markers of bone mineral metabolism in HIV infected patients taking protease inhibitor containing potent antiretroviral therapy. Antiviral Ther 2000; 5: S5-18. 
15.- Dusso A, Vidal M, Powderly W, Yarashesk K, Tebas P. Protease inhibitors inhibit in vitro conversion of 25 $(\mathrm{OH})$-vitamin D to 1,25 $(\mathrm{OH})$ 2-vitamin. Antiviral Ther 2000; 5 S15: 19.

16.- Carr A, Cooper DA. A randomized, multicenter study of protease inhibitor (PI) sustitution in aviremic patients with antiretroviral (ARV) lipodystrophy syndrome [abstract 205]. Program and Abstracts $7^{\text {th }}$ Conference on Retroviruses and Opportunistic
Infections. San Francisco California. www.retrocon ference.org

17.- Orwoll E, Ettinger M, Weiss S et al. Alendronate for the treatment of osteoporosis in men. N Eng J Med 2000343 (9): 604-10.

18.- Guaraldi G, Ventura P, Albuzza M, Orlando G, Bedini A, Esposito R. Alendronate treatment for osteoporosis in patients infected with Human Immunodeficiency Virus. Clin Infec Dis 2001, 33: 414-5.

Correspondencia a:

Marín Lasso Barreto

E-mail: zoalde@yahoo.com 